\section{Practically essential}

Aksel A. Bothner-By

Nuclear Magnetlc Resonance Spectroscopy, 2nd Edn. By Frank A. Bovey. Academic: 1988. Pp.653. \$49.95, £33.50.

ONE cannot be a chemist these days without knowing a good deal about nuclear magnetic resonance spectroscopy. Any current issue of a chemical or biochemical journal demonstrates that the technique is indispensable for establishing chemical structure, and today it is universally used for that purpose. Anybody who practises or wants to practise in this area has to become familiar with the theory and methods concerned, particularly as used for structural investigation, so that he may profit from this ubiquitous and powerful method.

In preparing the second edition of his book (the first appeared in 1969, since when NMR has evolved spectacularly), Dr Bovey set himself the task of making the methodology available to chemists who have not had a rigorous formal training in the quantum mechanics of particles with angular momentum. So there are

\section{RESEARCH REAGENTS FOR AMINO-SUGARS}

A series of novel basic polysaccharides purified from the culture fluid of $\mathrm{Pae}$ cilomyses sp. I-1 are manufactured by Higeta Shoyu Co., Ltd. and supplied by Funakoshi pharmaceutical Co., Ltd. exclusively.

$* \alpha \cdot 1,4$-POLYGALACTOSAMINE * GALACTOSAMINO-OLIGOSACCHARIDES * N-ACETYLGALACTOSAMINO-OLIGO. SACCHARIDES

$\alpha$-1, 4.POLYGALACTOSAMINE

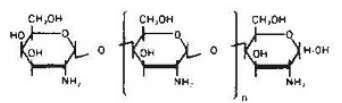

GALACTOSAMINO-OLIGOSACCHARIDES

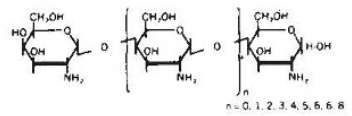

N-ACETYLGALACTOSAMINO-OLIGOSACCHARIDES

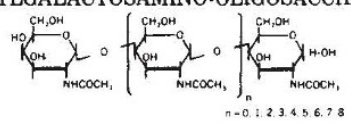

For further information contact

FUNAKOSHI PHARMACEUTICAL CO., LTD. 2.3 Surugadai, Kanda, Chiyoda-ku, Tokyo, Japan Telephone : Tokyo 03-293-2367

Ielefax : : 81-3-295-5545

Telex :I28489FUNA no hamiltonians, matrices (density or otherwise), commutators and the like to be found anywhere in the text.

Bovey has made a dedicated effort to present all the phenomena, interpretations, methods and empirical correlations in easily visualized terms. In many areas, this approach is gratifyingly successful students, whether undergraduate, graduate or grey-haired, will find his book a valuable guide to this field, with extensive maps, charts and advice, all in readily understandable form.

But there is an inevitable price to be paid for treating an obstinately quantum mechanical phenomenon in classical and non-mathematical terms. Occasionally, the classical picture will not fit with what is observed in the laboratory, and the results of experiments then appear enigmatic, to say the least. The powerful two-dimensional methods developed in the past few years are unintelligible except in quantum mechanical terms, yet they yield structural data of immense importance; heteronuclear double quantum coherence is an example. Treatment of this family of experiments is sparse or absent from the book, and almost necessarily so. The qualitative description of NMR phenomena is, in places, dangerously loose and could leave the reader stranded on the wrong path.

Probably the strongest feature is Bovey's presentation of practical data: tables of shifts, coupling constants, nuclear properties, spectral patterns, and structurespectra connections. These tables will certainly be valuable as guideposts in spectral interpretation, and will be of immediate use to those embarking on the NMR voyage of discovery.

Aksel A. Bothner-By is Professor of Chemistry and Acting Head of the Department of Chemistry at Carnegie Mellon University, Pittsburgh, Pennsylvania 15213, USA.

\section{There and back again}

\section{F.R. Harden Jones}

Dladromy in Fishes: Migrations Between Freshwater and Marine Environments. By Robert M. McDowall. Croom Helm (Chapman \& Hall), London/Timber Press, Portland, Oregon: 1988. Pp.308. £30, $\$ 47.95$.

DIADROMY describes migrations in which fish move between the sea and freshwater. Anadromous fish (such as salmon) move from the sea to breed in freshwater; catadromous fish (eels) move from freshwater to breed in the sea; and amphidromous fish (whitebait and gobies) move between the two domains for pleasures other than that of reproduction. The phenomenon is shown by more than 200 species from over 40 families: and if there be "a purpose in liquidity", then surely there must also be a purpose in diadromy.

Although there are several reviews on the subject, there has been no one volume which brings the facts together. Robert McDowall has set out to make good the need and he does it well. The book is logically structured, passing from definitions to the occurrence of the three forms of diadromy among fish, and so on, to details of life histories and their ecological significance. The author reviews the problems in establishing diadromous populations where they do not occur naturally, both the commercial and recreational importance of diadromous species, and concerns over those that are endangered.

The approach to the subject is truly global; a vast amount of information has been abstracted from the literature (there are over 600 references, going up to 1987) and is presented to the reader in a series of intelligible figures and tables. McDowall suggests (p.119) that the direction of migrations - anadromous at high latitudes and feeding at sea, catadromous at low latitudes and feeding in freshwater could be related to the observation that marine habitats are more productive at high latitudes and less so towards the tropics, whereas freshwater habitats have greatest productivity towards the tropics and less towards the poles. Right or wrong, this is a stimulating idea, and there are others.

I enjoyed the book and learned a great deal from it; it is unusually readable, and there is much here for the general reader.

Although semantic problems are dealt with carefully, it is disappointing to find no reference to John Kennedy's contributions to our understanding of the term migration. The index contains 700 itemized entries, which falls far short of that to be expected in a scholarly work of 300 pages: a string of page numbers is useless as a retrieval system for the serious student. There are also some curious errors, evidence of sloppy proof reading. A hyphen in Harden Jones is perhaps no more than one deserves, but to refer consistently to Professor A. D. Hasler as Haslar is inexcusable.

F. R. Harden Jones is Chief of the CSIRO Division of Fisheries, GPO Box 1538, Hobart, Tasmania 7001, Australia.

\section{New in paperback}

- Superconductors: The Breakthrough by Robert M. Hazen (Unwin Hyman, £5.99. For review see Nature 334, 479 (1988).

- Beyond the Laboratory: Scientists as Political Activists in 1930s America by Peter J. Kuznick (University of Chicago Press, \$14.95, £11.95). For review see Nature 332, 25 (1988). 